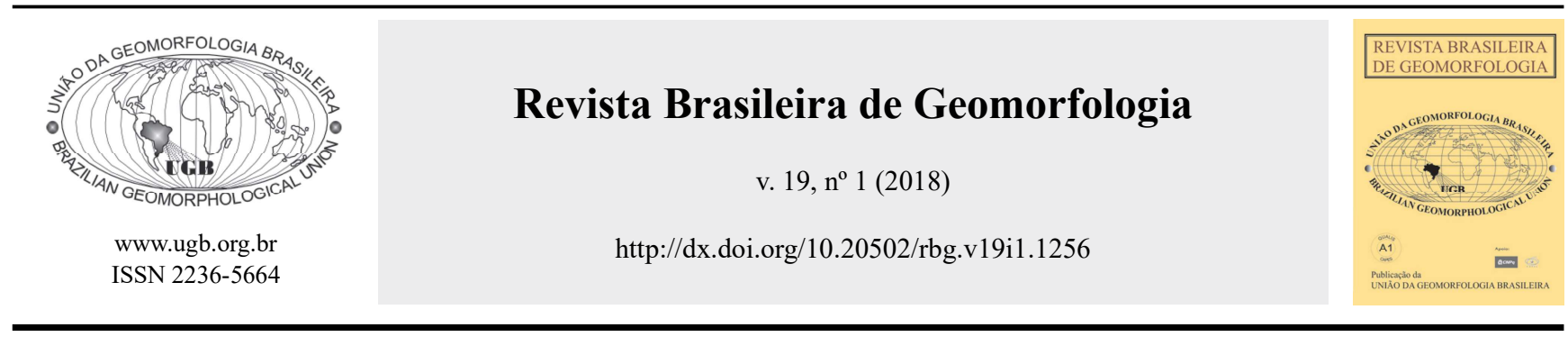

\title{
AVALIAÇÃO DA VARIAÇÃO DO FATOR DE SEGURANÇA COM O MODELO TRIGRS
}

\section{SAFETY FACTOR VARIATION ANALYSIS BY TRIGRS MODEL}

\author{
Fabrizio de Luiz Rosito Listo \\ Departamento de Ciências Geográficas, Universidade Federal de Pernambuco \\ Av. Acadêmico Hélio Ramos, s/n - Cidade Universitária, Recife, Pernambuco. CEP: 50.740-530, Brasil \\ Email:fabrizio.listo@ufpe.br
}

Maria Carolina Villaça Gomes

Departamento de Geografia, Universidade do Estado de Santa Catarina Av. Madre Benvenuta, 2007, Florianópolis, Santa Catarina. CEP: 88036-500. Brasil

Email: mcarolvg@yahoo.com.br

Bianca Carvalho Vieira

Departamento de Geografia, Universidade de São Paulo Av. Prof. Lineu Prestes, 338, São Paulo, São Paulo. CEP: 05508-000. Brasil

Email: biancacv@usp.br

\section{Informações sobre o Artigo}

Recebido (Received):

20/04/2017

Aceito (Accepted):

$01 / 11 / 2017$

\section{Palavras-chave:}

TRIGRS; Fator de Segurança; Escorregamentos translacionais rasos.

\section{Keywords:}

TRIGRS; Safety Factor;

Shallow landslides.

\section{Resumo:}

A modelagem matemática em base física desenvolvida para a previsão de áreas suscetíveis a escorregamentos tem como principal finalidade indicar os locais onde a estabilidade atinge valores críticos. O modelo TRIGRS, por sua vez, ainda oferece a possibilidade de identificação da profundidade onde o Fator de Segurança atinge valores inferiores a um, constituindo, então, superfícies hipotéticas de ruptura, informação importante para diversas finalidades. O objetivo deste trabalho foi avaliar a variação do Fator de Segurança em profundidade a partir dos resultados do modelo matemático TRIGRS, indicando-se uma possível superfície de ruptura dos escorregamentos ocorridos em março de 1967 em Caraguatatuba, Serra do Mar Paulista. Para atingir este objetivo foi gerado um cenário de suscetibilidade a partir de dados coletados in situ, que foram posteriormente validados utilizando-se um mapa de cicatrizes de escorregamentos. Os resultados apontaram uma superfície de ruptura entre $2,00 \mathrm{~m}$ e $2,50 \mathrm{~m}$. A identificação desta possível superfície de ruptura poderá ajudar num futuro monitoramento hidrológico e geotécnico, sobretudo para definir com precisão a localização da instalação de instrumentos, como sensores de umidade, por exemplo.

\footnotetext{
Abstract:

The mathematical modeling on physical base developed for the landslides susceptible areas forecast have the main purpose to indicate the places where
} 
the stability reaches critical values. The TRIGRS model, in turn, still offers the possibility of identifying the depth where the Safety Factor reaches values below one, constituting, therefore, hypothetical rupture surfaces, important information for several purposes. The objective of this work was to evaluate the Safety Factor variation in depth from the results of TRIGRS mathematical model, indicating possible landslides rupture surface occurred in March 1967 in Caraguatatuba city, Serra do Mar mountain ranges. To reach this objective, a susceptibility scenario was generated from data collected in the field, which were later validated using a landslide scars map. The results indicated a rupture surface between $2.00 \mathrm{~m}$ and $2.50 \mathrm{~m}$. The identification of this possible rupture surface may help in future hydrological and geotechnical monitoring, especially to define with accuracy the instruments installation location, such as moisture sensors, for example.

\section{Introdução}

Muitas pesquisas que buscam identificar áreas suscetíveis a escorregamentos rasos têm utilizado como método de previsão, os modelos matemáticos em bases físicas. Sua ampla utilização está associada à sua capacidade de gerar com rapidez e com precisão a localização de áreas instáveis, bem como à redução da subjetividade na análise de estabilidade do relevo, uma vez que o resultado é previsto de forma exata, baseados em noções matemáticas clássicas de relações exatamente previsíveis (CHORLEY, 1967; LISTO e VIEIRA; 2012, entre outros).

Estes modelos, cujo desenvolvimento é fundamentado em leis físicas naturais, baseiamse, normalmente, em modelos de estabilidade e hidrológicos, tendo como principais parâmetros de entrada, dados topográficos e propriedades físicas e mecânicas dos solos (SELBY, 1993; GUZZETI et al., 1999; van WESTEN, 2004). Os graus de suscetibilidade aos escorregamentos são quantificados em valores absolutos, geralmente, por meio do cálculo do Fator de Segurança (FS), que representa a razão entre a resultante das forças resistentes e a resultante das forças favoráveis ao movimento (TERLIEN et al.,1995).

Dentre os modelos, observa-se que alguns se destacam pelos resultados satisfatórios obtidos, como o SHALSTAB (Shallow Landslide Stability) (MONTGOMERY e DIETRICH, 1994), o SINMAP (Stability Index Mapping) (PACK et al., 1998) e o TRIGRS (Transient Rainfall Infiltration and Grid-Based Regional Slope-Stability) (BAUM et al., 2002). Enquanto o SHALSTAB e o SINMAP se baseiam em modelos de estabilidade associados a um modelo hidrológico steadystate, o TRIGRS calcula a variação do FS em diferentes profundidades, conforme a mudança na pressão da água no solo ao longo de um evento pluviométrico, ou seja, é transiente (BAUM et al., 2002).
Esta diferença do TRIGRS em relação aos demais modelos está associada ao modelo hidrológico utilizado, que assume a existência de uma frente de infiltração, considerando-se somente o fluxo vertical, normal à superfície. Além dos parâmetros topográficos, mecânicos e hidrológicos dos solos, são consideradas as características dos eventos pluviométricos e sua variação ao longo do tempo, como a intensidade dos eventos e a duração acumulada destes.

No Brasil, os modelos em bases físicas foram aplicados, em sua maioria, na Serra do Mar. As pesquisas realizadas utilizaram o SHALSTAB (GUIMARÃES et al., 2003; FERNANDES et al., 2004; RABACO, 2005; GOMES, 2006; VIEIRA et al., 2010, MICHEL et al., 2014; VIEIRAe RAMOS, 2015, entre outros), o SINMAP (LOPES, 2006; SILVA et al., 2012; MICHEL et al., 2014; NERY e VIEIRA, 2015) e o TRIGRS (RABACO, 2005; VIEIRA et al., 2010; LISTO e VIEIRA, 2015). As aplicações dos modelos também diferiram no que se refere à fonte dos parâmetros de entrada. Enquanto parte dos trabalhos utilizou dados disponíveis na literatura, naqueles em que foram empregados dados coletados in situ, o desempenho foi superior, considerando a maior precisão entre as áreas classificadas como instáveis e a ocorrência de escorregamentos pretéritos (ex. LISTO e VIEIRA, 2015).

Embora sejam satisfatórios os resultados obtidos a partir destes modelos, destaca-se que o TRIGRS ainda fornece outro resultado - a variação em profundidade do Fator de Segurança. A existência de profundidades onde o FS é reduzido até que seja inferior a 1 poderia indicar uma superfície hipotética de ruptura, informação importante para diversas finalidades. Dentre elas, pode-se citar: (i) a compreensão dos mecanismos de instabilização; (ii) a estimativa de volume de material mobilizado por um evento; e (iii) a definição de locais preferenciais para amostragem, de levantamentos in situ e de monitoramentos em tempo real das propriedades hidrológicas dos solos. 
Trabalhos desenvolvidos com o objetivo de caracterizar as propriedades físicas, mecânicas e hidrológicas dos perfis de alteração na Serra do Mar em Caraguatatuba-SP mostraram que há descontinuidades mecânicas e hidráulicas nos materiais, o que poderia constituir planos preferenciais para a ocorrência dos escorregamentos rasos (VIEIRA e FERREIRA, 2015; GOMES e VIEIRA, 2016). Mesmo se tratando de levantamentos pontuais, os dados coletados in situ são essenciais para sua utilização nos modelos. Desta forma, o objetivo deste trabalho foi avaliar a variação do Fator de Segurança em profundidade a partir dos resultados do modelo TRIGRS, na bacia do rio Guaxinduba em Caraguatatuba-SP, indicando-se uma possível superfície de ruptura, e sua relação com parâmetros mecânicos e hidrológicos coletados in situ.

\section{Bacia do rio Guaxinduba, Serra do Mar Paulista - Histórico de Escorregamentos e Caracterização}

Situada em Caraguatatuba no Litoral Norte de São Paulo na escarpa da Serra do Mar Paulista, a Bacia do rio Guaxinduba (Figura 1) foi uma das áreas onde os escorregamentos ocorreram em maior concentração quando estes atingiram amplamente esta unidade geomorfológica em março de 1967. As encostas que foram afetadas pelos escorregamentos encontravamse com cobertura vegetal bem preservada, tendo sido submetidas a chuvas intensas e prolongadas. $\mathrm{O}$ volume de chuva registrado ao longo do verão de 1966/67 foi acima do normal, culminando com o episódio dos dias 17 e 18 de março. No mês de março, foram registrados $945,6 \mathrm{~mm}$, sendo que apenas nos dias 17 e 18 choveram cerca de $600 \mathrm{~mm}$ (CRUZ, 1974), correspondendo a cerca de $45 \%$ do total pluviométrico anual médio da região (WOLLE, 1986).

Escorregamentos coletivos e corridas de detritos atingiram principalmente as bacias dos rios Guaxinduba, Santo Antônio, Canivetal, Aldeia e Pau D'Alho, cujas cabeceiras se encontram nas isoietas de maior valor deste evento pluviométrico. Os escorregamentos se concentraram nas encostas acima de $20^{\circ} \mathrm{e}$, em sua maioria, a ruptura ocorreu no contato entre solos eluviais maduros e o saprólito sobrejacente (CRUZ, 1974), tendo sido observadas cicatrizes com até $5 \mathrm{~m}$ de profundidade. $\mathrm{O}$ grande volume de material coluvionar mobilizado, estimado em aproximadamente $30.000 .000 \mathrm{t}$ conforme Fúlfaro et al. (1976), foi transportado por corridas de detritos até a área urbana de Caraguatatuba, na planície costeira, causando, ao menos, 436 vítimas fatais e deixando 3.000 pessoas desalojadas ( $20 \%$ da população).

No setor da Serra do Mar correspondente à Bacia do Guaxinduba, predominam as variações litológicas cristalinas, como os migmatitos, os granitoides gnáissicos, os paragnaisses, os gnaisses paraluminosos e os granitos-gnaisses (ALMEIDA e CARNEIRO, 1998; PERROTA et al., 2005). Segundo Cruz (1974), os paragnaisses sustentam os morros mais elevados e os divisores de água, enquanto os litotipos mais suscetíveis ao intemperismo ou os diversos lineamentos ortogonais existentes condicionam a formação dos relevos mais baixos e dos sistemas fluviais.

A bacia possui área de $24 \mathrm{~km}^{2}$, amplitude altimétrica de aproximadamente $1.000 \mathrm{~m}$, ângulos superiores a $30^{\circ}$ no setor escarpado, bem como outras características morfométricas e morfológicas que lhe conferem um elevado potencial morfodinâmico. Em decorrência das altas taxas de morfogênese, as encostas são recobertas por solos eluviais e por depósitos de colúvios. Nas áreas escarpadas predominam, majoritariamente, os cambissolos e, em menor quantidade, os neossolos litólitos e os depósitos coluvionares e de tálus (colúvio grosso) (CRUZ, 1974). Para a autora, os solos eluviais têm matriz silto-arenosa, geralmente com espessuras entre $0,5 \mathrm{~m}$ a $2 \mathrm{~m}$, ao passo que os saprólitos apresentam grandes espessuras (até os $80 \mathrm{~m}$ ), constituído de um material pouco consistente, com preservação da estrutura do material parental, como bandamentos e foliações, além de apresentarem-se muito fraturados, favorecendo a percolação da água no interior do maciço.

A caracterização físico-mecânica dos solos próximos a cicatrizes de 1967 realizada por Vieira et al. (2015) mostrou elevados percentuais das frações de areia (cerca de $80 \%$ ), destacando as areias siltosas pouco argilosas e siltes pouco argilosos. Os baixos teores de argila encontram-se concentrados em horizontes mais superficiais $(\sim 1 \mathrm{~m})$, mas estas são constituídas de argilominerais não plásticos ou inativos. No que se refere à porosidade total, verificou-se valores médios em torno de $65 \%$, com predomínio de microporos, que estão diretamente relacionados às variações do teor de argila. Quanto às propriedades mecânicas, o ângulo de atrito interno dos materiais superficiais variou entre $25^{\circ}$ a $29^{\circ}$ e nos materiais sotopostos, de cerca de $32^{\circ}$ a $36^{\circ}$, enquanto os valores de coesão variaram entre 0 $\mathrm{kPa}$ e $11 \mathrm{kPa}$. A condutividade hidráulica saturada $\left(K_{\text {sat }}\right)$ 
destes solos, de acordo com Gomes e Vieira (2016), varia entre as ordens de grandeza $10^{-4} \mathrm{~m} / \mathrm{s}$ a $10^{-7} \mathrm{~m} / \mathrm{s}$, principalmente $10^{-5} \mathrm{~m} / \mathrm{s}$ e $10^{-6} \mathrm{~m} / \mathrm{s}$, tendo sido observadas descontinuidades hidráulicas entre $1 \mathrm{~m}$ e $2,5 \mathrm{~m}$, em que há a redução em até 100 vezes da $K_{\text {sat }}$ bem como a tendência de aumento com a profundidade (até $5 \mathrm{~m}$ ).

Segundo Cruz (1974), os índices pluviométricos dos anos habituais variam entre $1.750 \mathrm{~mm}$ e 2.000 $\mathrm{mm}$, mas, a condição favorável à chegada de umidade associada à Zona de Convergência do Atlântico Sul (ZCAS) bem como de sistemas frontais provenientes do Sul (Frente Polar Atlântica), levam à ocorrência de anos muito chuvosos, podendo alcançar até $4.000 \mathrm{~mm}$ na região (SANTOS, 2015).
A umidade elevada é um fator fundamental para o desenvolvimento da Floresta Ombrófila Densa, sobretudo o seu subtipo Montana, que recobre grande parte do setor escarpado da Serra do Mar. Embora apresente um alto grau de preservação em função da existência de uma Unidade de Conservação que a protege, sua existência não assegura a estabilidade das encostas. As funções mecânicas e hidrológicas parecem favorecer a estabilidade, no entanto, em situações de índices pluviométricos extremos, mesmo as áreas preservadas não estão imunes aos escorregamentos, o que pôde ser verificado no evento de 1967, quando estes foram majoritariamente deflagrados em encostas isentas de ocupação antrópica.

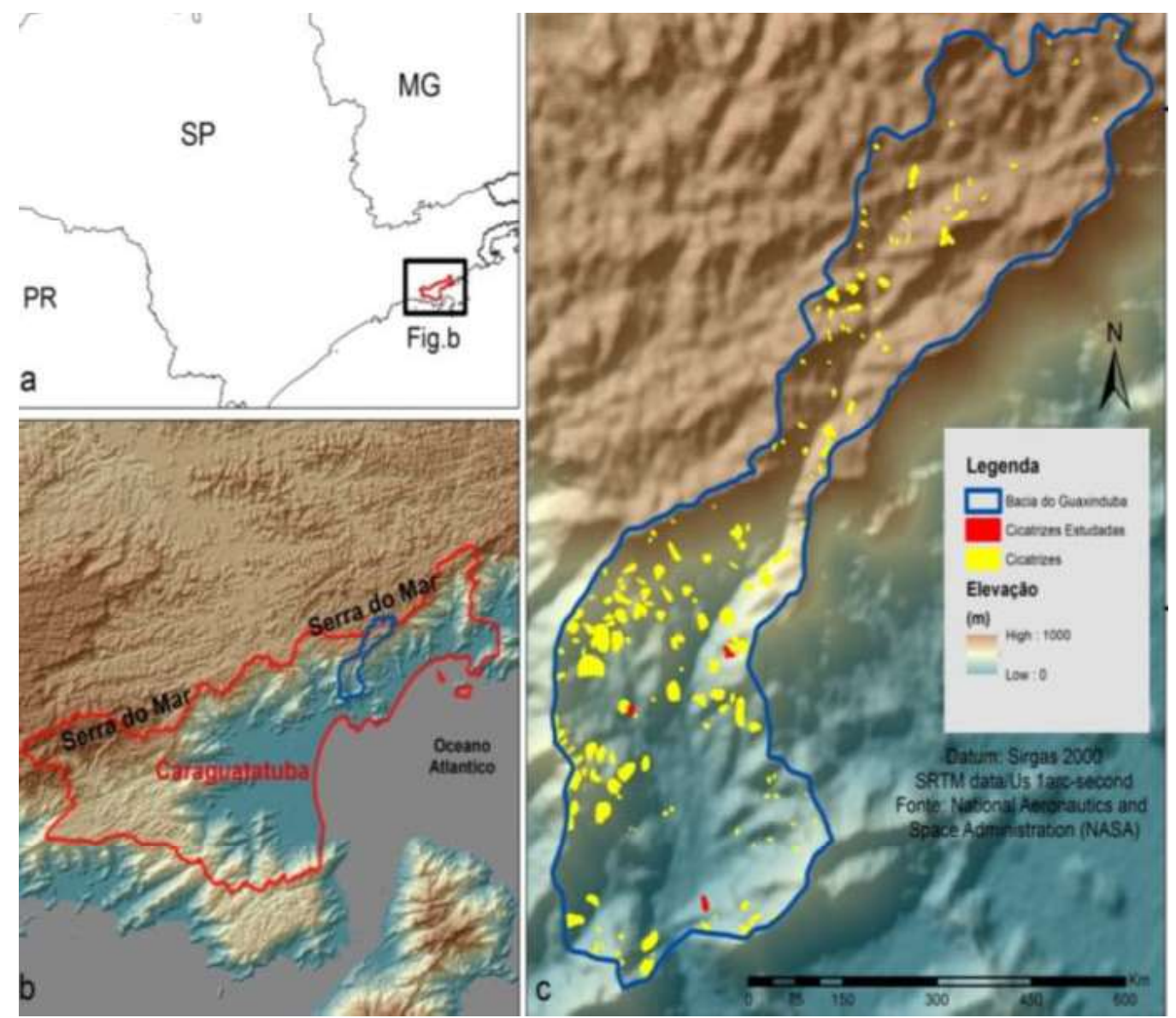

Figura 1 - Localização da Bacia do Guaxinduba no contexto do Estado de São Paulo (a) e do município de Caraguatatuba (b), na qual são demonstradas as cicatrizes de escorregamentos do evento de 1967 (c).

\section{Materiais e Métodos}

\section{Cenário de suscetibilidade e inventário de escorregamentos (validação do cenário)}

O TRIGRS avalia a suscetibilidade aos escorregamentos translacionais rasos utilizando parâmetros topográficos; pluviométricos; mecânicos e hidrológicos. Dessa forma, foi gerado um cenário de suscetibilidade, posteriormente validado utilizando-se um mapa de cicatrizes dos escorregamentos ocorridos em 1967.

Os parâmetros topográficos foram obtidos a partir de um Modelo Digital do Terreno com resolução de 
$25 \mathrm{~m}^{2}$, fornecido pela Emplasa (2011) e elaborado por meio de tecnologia LiDAR (Light Detection and Ranging), a qual se baseia em nuvens de pontos coletados.

Quanto aos parâmetros pluviométricos (tempo inicial das chuvas, sua intensidade e a duração acumulada dos eventos), estes foram capturados das estações pluviométricas do Sistema SIGRH/DAEE (Sistema Integrado de Gerenciamento de Recursos Hídricos do Estado de São Paulo/Departamento de Águas e Energia Elétrica). Foi considerada a precisão dos dados (ex. inexistência de falhas); a proximidade da estação em relação à bacia de estudo desta pesquisa e as séries temporais referentes aos dias de ocorrência dos escorregamentos de 1967 (chuvas antecedentes e precipitação durante os eventos). A partir destes critérios, as estações utilizadas neste trabalho foram a E2-042 (altitude de $20 \mathrm{~m}$ e coordenadas $23^{\circ} 35^{\prime} 00^{\prime \prime} \mathrm{S}$; $45^{\circ} 27^{\prime} 00^{\prime \prime} \mathrm{O}$ ); E2-043 (altitude de $200 \mathrm{~m}$ e coordenadas $23^{\circ} 37^{\prime} 00^{\prime \prime} \mathrm{S}$; 4526'00”O) e E2-046 (altitude de $20 \mathrm{~m}$

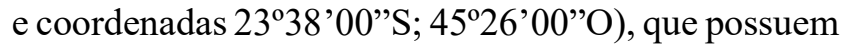
dados de chuvas diárias acumuladas $(\mathrm{mm} / \mathrm{dia})$ e chuvas mensais acumuladas ( $\mathrm{mm} / \mathrm{mês})$ para o período de março de 1967 e são próximos à bacia (Figura 2).

Por fim, os valores mecânicos (coesão, peso especifico do solo e ângulo de atrito) e hidrológicos (condutividade hidráulica saturada) foram coletados in situ por Ferreira (2013) e Gomes (2012), respectivamente, em três cicatrizes na bacias $(\mathrm{C} 1$, C2 e C3). A média aritmética dos valores obtidos nos levantamentos nestas cicatrizes foi adotada como entrada dos parâmetros mecânicos e hidrológicos no TRIGRS (Figura 3 e Tabela 1).

A partir de 37 amostras deformadas e 24 amostras indeformadas, Ferreira (2013) realizou ensaios de granulometria, densidade, porosidade total, macroporosidade e microporosidade. Seis blocos indeformados foram utilizados nos ensaios de resistência ao cisalhamento direto para determinação da coesão e do ângulo de atrito interno, tendo sido coletados nas seguintes profundidades: $\mathrm{C} 1(0,45 \mathrm{~m}$ e $1,45 \mathrm{~m})$; C2 $(0,60 \mathrm{~m}$ e $2 \mathrm{~m})$ e $\mathrm{C} 3(0,45 \mathrm{~m}$ e $2 \mathrm{~m})$. A $K_{\text {sat }}$ foi mensurada nestas mesmas cicatrizes com o Permeâmetro de Guelph (GOMES, 2012), nas profundidades de $0,25 \mathrm{~m} ; 0,50 \mathrm{~m} ; 1,00 \mathrm{~m} ; 1,50 \mathrm{~m}$; $2,00 \mathrm{~m} \mathrm{e} 2,50 \mathrm{~m}$.
Estas cicatrizes foram selecionadas em razão dos seguintes critérios: (a) acesso às áreas atingidas; (b) relevância das características físicas e bióticas da Serra do Mar e (c) inexistência de interferência antropogênica no terreno (ex. taludes artificiais de corte). Foram abertos três perfis em cada cicatriz (no topo, na lateral e no seu interior), até a profundidade de 2,50 m, aproximadamente. A escolha dos locais para abertura dos perfis se deve ao fato de se presumir que o manto de alteração removido tem semelhanças ao de montante e, sobrepondo o perfil do topo ao do centro da cicatriz, torna-se possível reconstituir o perfil de alteração anterior à ruptura (retroanálise).

Os valores do Default do modelo foram utilizados para alguns dados, como a altura inicial do lençol freático $(d)$, a taxa de infiltração inicial $\left(I_{L T}\right)$ e a difusividade hidráulica $\left(D_{0}\right)$, uma vez que não há dados coletados in situ e, para estes casos, são considerados eficientes.

Os cenários foram validados a partir de uma análise de sobreposição com o inventário de cicatrizes dos escorregamentos de 1967 elaborado por Vieira e Ramos (2015). Tais autores identificaram 188 cicatrizes por meio de interpretação de fotografias áreas ortorretificadas disponibilizadas, gratuitamente, pelo Instituto Florestal de São Paulo (IF/SP), do ano de 2000 com escala de 1:5.000. Para o mapeamento das cicatrizes, foram consideradas as diferenças de cor e de textura e seu aspecto alongado na fotografia aérea, destacando-se que foram consideradas somente as áreas de ruptura, sendo excluídas suas áreas de arraste e de depósito coluvionar.

A validação, a partir da combinação do mapa de suscetibilidade com o mapa de cicatrizes, foi realizada utilizando-se os seguintes índices estatísticos: (a) Concentração de Cicatrizes (CC): relação entre o número de pixels, de cada classe, atingidas pelas cicatrizes e o total de pixels atingidas na bacia e (b) Potencial de Escorregamentos (PE): relação entre o número de pixels, de cada classe, atingidas pelas cicatrizes e o total de pixels dessa mesma classe. Foi utilizado também o índice FD (Frequência de Distribuição), que indica a distribuição percentual das classes de suscetibilidade calculadas pelo modelo (relação entre o total de cada classe e o valor total de pixels da bacia). 
Listo F. L. R. et al.

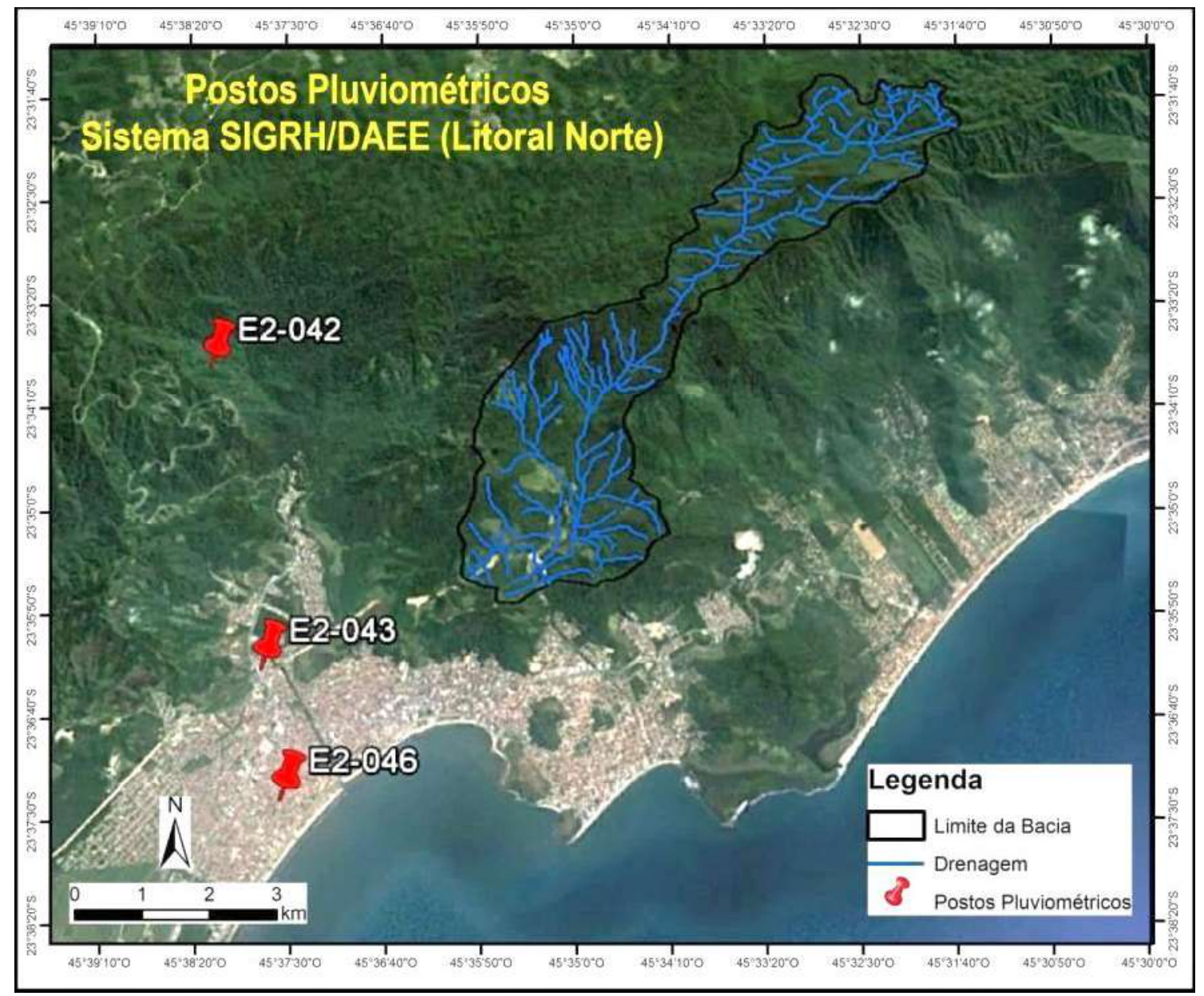

Figura 2 - Localização dos três postos pluviométricos em funcionamento em 1967, próximos a Bacia do Guaxinduba. Fonte: Organizado pelos autores a partir de dados do Sistema SIGRH/DAEE (2015).

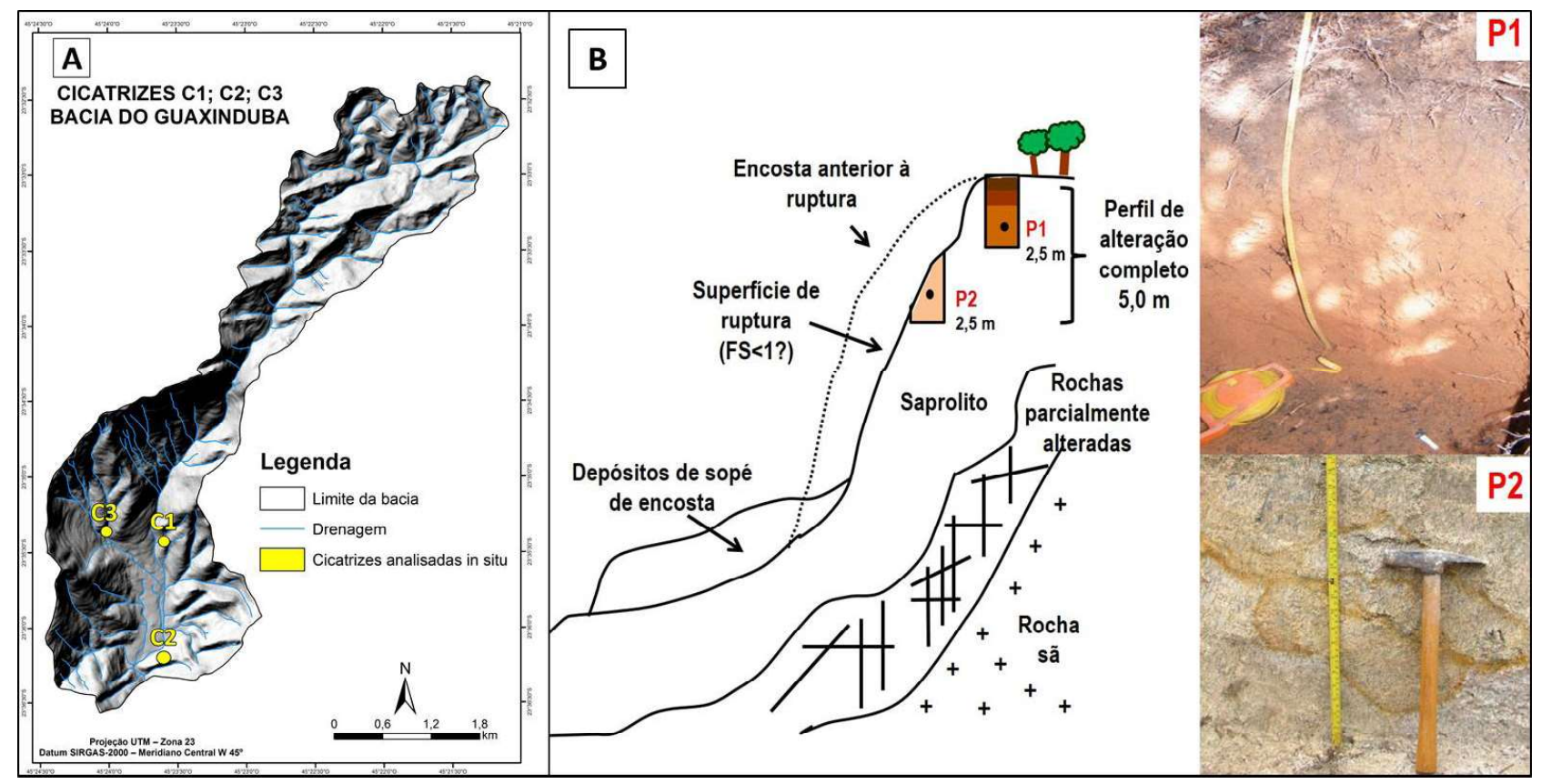

Figura 3 - (A) Localização das cicatrizes dos escorregamentos (C1; C2 e C3) onde foram feitas as coletas de amostras de solos; (B) Esquema da localização dos pontos de amostragem e de levantamentos in situ, destacando-se como foram obtidos os valores para um perfil de alteração com $5,0 \mathrm{~m}$. 
Tabela 1: Valores referentes aos parâmetros mecânicos e hidrológicos utilizados no modelo TRIGRS.

\begin{tabular}{c|c}
\hline PARÂMETROS [unidade] & TRIGRS \\
\hline Coesão do solo $(c)[\mathrm{kPa}]$ & $4^{*}$ \\
\hline Densidade do solo $\left(\rho_{s}\right)\left[\mathrm{kN} / \mathrm{m}^{3}\right]$ & $15^{*}$ \\
\hline Espessura máx. do solo $\left(Z_{\max }\right)[\mathrm{m}]$ & $3 *$ \\
\hline Ângulo de atrito interno $(\phi)\left[{ }^{\circ}\right]$ & $34^{*}$ \\
\hline Alt. Ini. do lençol freático $(d)[\mathrm{m}]$ & 3 \\
\hline Taxa de infiltração inicial $I_{L T}[\mathrm{~m} / \mathrm{s}]$ & $1,0 \times 10^{-9}$ \\
\hline Difusividade hidráulica $D_{0}\left[\mathrm{~m}^{2} / \mathrm{s}\right]$ & $5,5 \times 10^{-4}$ \\
\hline Cond. hidr. saturada vert. $\left(K_{s a t}\right)[\mathrm{m} / \mathrm{s}]$ & $1,0 \times 10^{-5 *}$
\end{tabular}

*Valores referentes à média aritmética dos parâmetros levantados nas três cicatrizes (C1; C2 e C3). Fonte: autores.

\section{Variação do Fator de Segurança}

Além do mapa de suscetibilidade, o TRIGRS produz, para um mesmo cenário, os dados de escoamento superficial (runoff) para uma determinada chuva; o FS mínimo para cada grid e a profundidade do solo com FS mínimo para ruptura ( $\mathrm{FS}<1$ ), que neste caso pode indicar uma possível superfície de ruptura. Em outras palavras, para cada unidade do grid do mapa de suscetibilidade, o modelo calcula a variação do FS em profundidade e quando este atingir um valor crítico de estabilidade, ou seja, $\mathrm{FS}<1$, pode-se admitir que exatamente naquela profundidade ocorreu a zona de ruptura do escorregamento.

Com base nisso, para a análise do Fator de Segurança em profundidade, foi escolhida uma área teste da bacia (sub-bacia a oeste) com diferentes graus de suscetibilidade e presença de cicatrizes para esta avaliação, inclusive a $\mathrm{C} 3$, investigadas por Gomes (2012) e Ferreira (2013) in situ (Figura 4). Dessa forma, foi possível verificar: (i) em qual profundidade do solo ocorreu FS $<1$ e (ii) uma relação mais específica desta profundidade com os dados mecânicos e hidrológicos de entrada do modelo, sobretudo, coesão (c), ângulo de atrito $(\phi)$ e condutividade hidráulica saturada $\left(k_{s a t}\right)$.

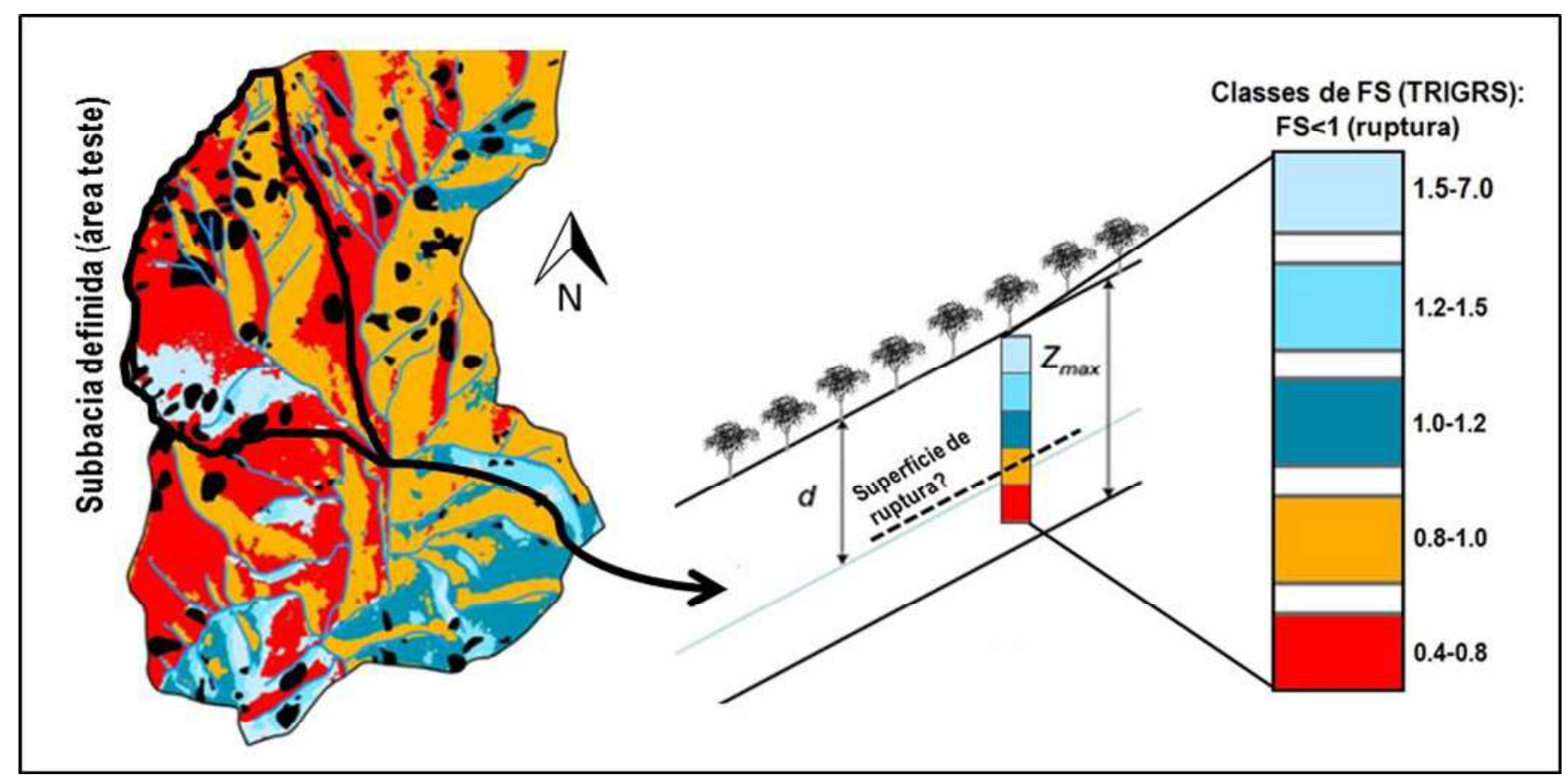

Figura 4 - À esquerda, sub-bacia teste para avaliação da variação do FS em profundidade, selecionada em função dos diferentes graus de suscetibilidade e da presença de cicatrizes (inclusive a cicatriz C3, investigada por Gomes, 2012 e Ferreira, 2013 in situ). À direita, modelo hipotético exemplificando a profundidade do solo (Zmax) em que pode ocorrer a ruptura, ou seja, na qual o FS<1 é calculado pelo modelo conforme os parâmetros de entrada, sobretudo chuva e altura inicial do lençol freático. 


\section{Resultados e Discussões}

\section{Modelo TRIGRS}

O modelo apresentou, em relação ao índice FD (Figura 5), 71\% de áreas previstas em classes de instabilidade $(\mathrm{FS} \leq 1)$. Quanto à Concentração de Cicatrizes (CC), houve um maior percentual (aproximadamente 37\%) na classe de maior instabilidade do TRIGRS (FS entre 0,4 e 0,8); seguida de $30 \%$ na segunda classe mais instável, FS entre 0,8 e 1,0 (Figura 5). Assim, ao se considerar o somatório das classes de instabilidade do modelo, foi possível verificar um alto percentual de acerto (67\%). Quanto ao Potencial de Escorregamentos (PE), as classes instáveis apresentaram um percentual de $7 \%$ e as classes estáveis, somente 2,2\% (Figura 5), estando, portanto, em concordância com as áreas previstas como de instabilidade pelo TRIGRS e as cicatrizes dos escorregamentos.

Tais valores podem ser considerados altos e satisfatórios em comparação aos mesmos índices encontrados na literatura em trabalhos nacionais e internacionais que também utilizaram o TRIGRS (Tabela 2). Crosta e Frattini (2003), por exemplo, previram somente $17,5 \%$ de áreas instáveis em uma região montanhosa ao norte da Itália, mas que concentraram $73 \%$ do total de cicatrizes dos escorregamentos (CC). Salciarini et al. (2006) utilizaram o modelo na região de Umbria, também na Itália, com parâmetros mecânicos e hidrológicos obtidos em in situ e muito parecidos aos desta pesquisa (ex. $Z_{\max }=2 \mathrm{~m} ; \phi=33^{\circ}$ e $c=4$ $k P a)$, e do conjunto de 42 cicatrizes mapeadas, 33 foram previstas pelo modelo, apresentando uma $\mathrm{CC}$ de aproximadamente $78 \%$. Vieira et al. (2010) utilizaram o TRIGRS na Bacia do Rio Copebrás (Cubatão, Serra do Mar Paulista) e obtiveram o mesmo valor de CC deste trabalho, ou seja, 67\%. Por fim, Kin et al. (2013) aplicaram o modelo em Bonghwa (Coréia do Sul), mas obtiveram uma CC de apenas $30 \%$, provavelmente pelo alto valor de coesão do solo utilizado $(21,8 \mathrm{kPa})$, indicando que o modelo é, conforme já comprovado, bastante sensível a coesão do solo (Tabela 2).

Neste trabalho, a classe mais instável $(0,4$ e 0,8$)$ concentrou-se predominantemente nas sub-bacias a oeste e a sudoeste e em menor quantidade, a noroeste da bacia (Figura 5). A sudoeste e a oeste da bacia foram verificadas declividades superiores a $30^{\circ}$ e $40^{\circ}$, isto é, encostas de forte gradiente, no entanto, a noroeste ocorreram declives mais suaves $\left(\leq 20^{\circ}\right)$. As classes mais instáveis coincidiram com as sub-bacias de maior valor de área de contribuição, confirmando a influência das taxas de maior saturação do solo na determinação da instabilidade. Por fim, com relação às formas das encostas, houve uma localização de áreas instáveis de forma mais homogênea com as feições côncavas, retilíneas e convexas, dificultando a correlação das curvaturas com as áreas de maior instabilidade (Figura 5).

\section{Variação do FS conforme a profundidade do solo}

Os resultados para a sub-bacia selecionada (conforme a Figura 4) apontaram uma superfície de ruptura entre 2,00 m e 2,50 m (Figura 6). Ao analisar em conjunto este intervalo de profundidade com os parâmetros de entrada do TRIGRS, sobretudo com a $K_{\text {sat }}$, $c$ e $\phi$, observou-se que nas três cicatrizes a profundidade mais próxima de análise destes parâmetros equivale a 2,0 $\mathrm{m}$. Nesta profundidade, observou-se para $\mathrm{C} 1, c=1,19$ $\mathrm{kPa} ; \phi=31,9^{\circ}$ e $k_{\text {sat }}=10^{-4} \mathrm{~m} / \mathrm{s} ;$ para C2, $c=11,05 \mathrm{kPa} ; \phi=$ $36,9^{\circ}$ e $k_{\text {sat }}=10^{-5} \mathrm{~m} / \mathrm{s} \mathrm{e}$, para C $3, c=0 \mathrm{kPa} ; \phi=33,5^{\circ}$ e $k_{\text {sat }}$ $=10^{-5} \mathrm{~m} / \mathrm{s}$ (Tabela 3 ) (GOMES, 2012; FERREIRA, 2013).

Em relação à granulometria dos solos nas cicatrizes, Vieira et al. (2015) verificaram que até aproximadamente $1 \mathrm{~m}$ de profundidade há um aumento do percentual de silte e argila, esta última apresentando baixa atividade ou inatividade. A partir de $1 \mathrm{~m}$, há um aumento progressivo de areia, alcançando em torno de $80 \%$ aos $2 \mathrm{~m}$. Esta distribuição granulométrica pode levar à instabilização das encostas a partir de dois mecanismos: (i) o desenvolvimento de poropressões positivas onde há maior concentração de silte e argila e (ii) o elevado teor de areia permite com que a frente de infiltração avance a maiores profundidades, podendo, durante os eventos pluviométricos intensos e prolongados, levar à redução dos parâmetros de resistência dos materiais, como a coesão aparente.

Embora os valores de $K_{\text {sat }}$ obtidos por Gomes e Vieira (2016) não tenham apresentado grande amplitude $\left(10^{-6} \mathrm{~m} / \mathrm{s}\right.$ a $\left.10^{-4} \mathrm{~m} / \mathrm{s}\right)$, foram identificadas descontinuidades hidráulicas significativas entre $1 \mathrm{~m} \mathrm{e}$ $2,5 \mathrm{~m}$, em que a $K_{\text {sat }}$ chegou a ser reduzida em até 100 vezes em apenas $0,50 \mathrm{~cm}$ do solo. Ainda que o TRIGRS não considere a variação vertical das propriedades dos solos, observa-se que houve semelhança entre a superfície hipotética de ruptura e uma forte redução da $K_{\text {sat }}$ (Figura 6). Esta coincidência pode indicar que, na realidade, nestas profundidades, o FS poderia atingir um valor abaixo de $1 \mathrm{em}$ um intervalo de tempo menor, ou que, para um mesmo acumulado de chuva, seu valor seja ainda menor, tornando a instabilidade maior. 


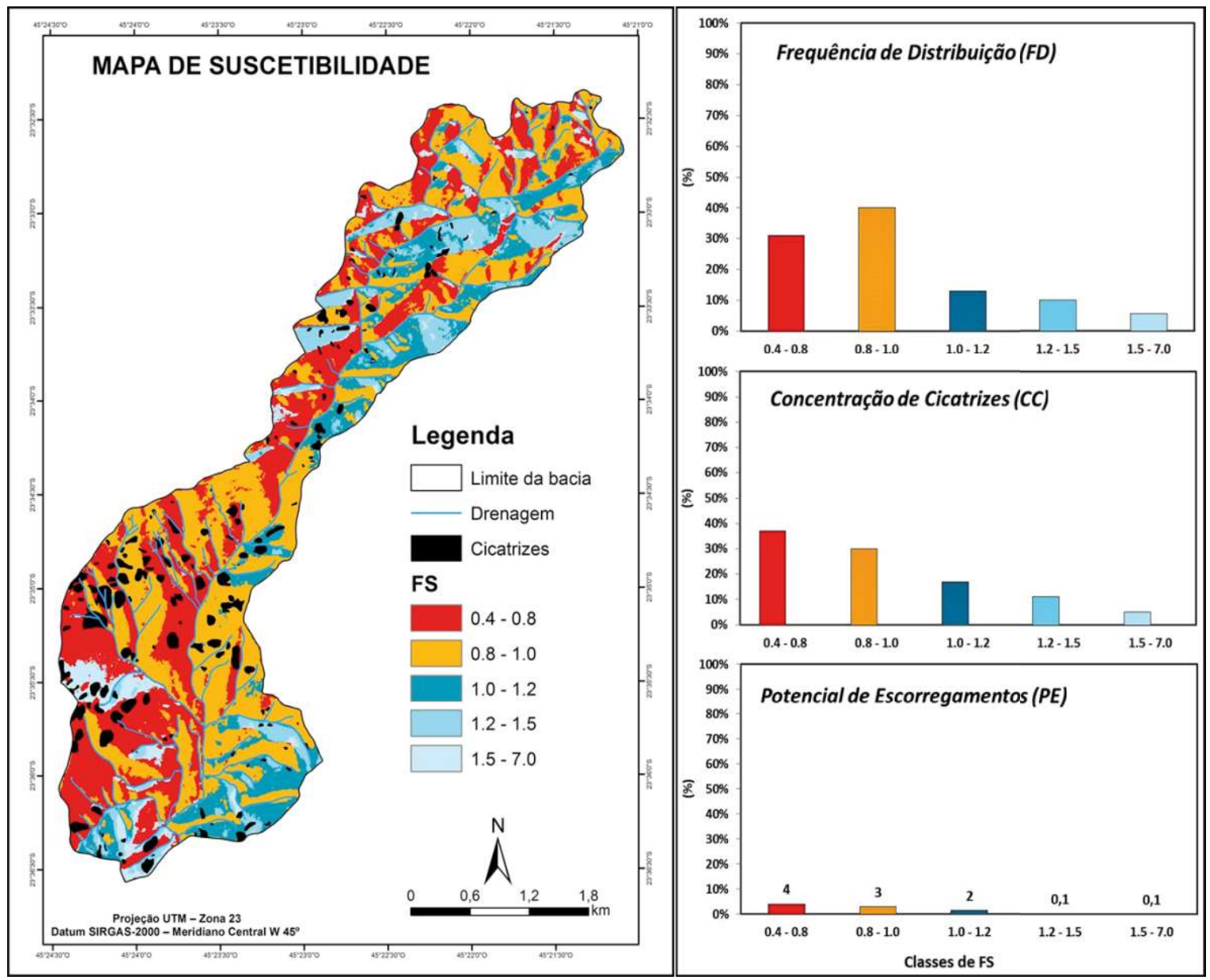

Figura 5 - Mapa de suscetibilidade gerado pelo modelo TRIGRS e indices de FD; CC e PE. Parâmetros utilizados: $c=4 \mathrm{kPa} ; \rho_{\mathrm{s}}=15 \mathrm{kN} /$ $\mathrm{m}^{3} ; Z_{\max }=3 \mathrm{~m} ; \phi=34^{\circ} ; d=3 \mathrm{~m} ; I_{L T}=1,0 \times 10^{-9} \mathrm{~m} / \mathrm{s} ; D_{0}=5,5 \times 10^{-4} \mathrm{~m}^{2} / \mathrm{s}$ e $K_{\text {sat }}=1,0 \times 10^{-5} \mathrm{~m} / \mathrm{s}$.

Tabela 2: Percentuais de acerto calculados a partir do índice CC (Concentração de Cicatrizes) em outros trabalhos que também utilizaram o Modelo TRIGRS.

\begin{tabular}{c|c|c|c}
\hline Autor(es) & Área de aplicação & CC (\%) & Observações \\
\hline Crosta e Frattini (2003) & Norte da Itália & 73 & Áreas instáveis: $17,5 \%$ \\
\hline Salciarini et al. (2006) & Umbria (Itália) & 78 & $\begin{array}{c}\text { Parâmetros de entrada semelhante } \\
\text { aos utilizados neste trabalho } \\
\left(Z_{\max }=2 \mathrm{~m} ; \phi=33^{\circ} \text { e c }=4 \mathrm{kPa}\right)\end{array}$ \\
\hline Vieira et al. (2010) & $\begin{array}{c}\text { Bacia da Copebrás } \\
\text { (Cubatão-SP) }\end{array}$ & 67 & $\begin{array}{c}\text { Mesmo valor de CC em relação ao } \\
\text { obtido neste trabalho }\end{array}$ \\
\hline Kin et al. (2013) & $\begin{array}{c}\text { Bonghwa } \\
\text { (Coréia do Sul) }\end{array}$ & 30 & $\begin{array}{c}\text { Coesão elevada: } 21,8 \mathrm{kPa} \\
\text { Parâmetros mecânicos } \boldsymbol{e} \\
\text { Este trabalhológicos levantados in situ }\end{array}$ \\
\hline
\end{tabular}

Fonte: organizado pelos autores.

A profundidade da superfície de ruptura indicada pelo TRIGRS neste trabalho $(2 \mathrm{~m}$ a $2,5 \mathrm{~m})$ está de acordo com a faixa de valores considerados comuns para escorregamentos translacionais rasos (entre $0,5 \mathrm{~m}$ e $5 \mathrm{~m}$ ) conforme classificações de diversas referências, tais como Augusto Filho (1992); Selby (1993); Fernandes e Amaral (1996); entre outros. Entretanto, muitos trabalhos, tais como Vargas et al. (1986); 
Wolle (1988); Wolle e Carvalho (1989); Campos et al. (1992); Wolle e Carvalho (1994) e Mendes (2008) apontaram que, especificamente para a Serra do Mar, os planos de ruptura são comuns entre $1,5 \mathrm{~m}$ e $2 \mathrm{~m}$, acima da superfície potencial de ruptura identificada nesta pesquisa (Tabela 4). Wolle e Carvalho (1989), por exemplo, afirmaram que as rupturas na Serra de Cubatão em 1985 ocorreram no contato entre os depósitos coluvionares e o saprólito, a $1 \mathrm{~m}$ ou pouco mais de profundidade (Tabela 4).

Por outro lado, Cruz (1979) observou que na Serra de Caraguatatuba, em 1967, os escorregamentos atingiram profundidades que alcançaram até os $5 \mathrm{~m}$, enquanto Silveira et al. (2012) afirmaram que os escorregamentos que atingiram a bacia do rio Jacareí, na Serra do Mar Paranaense, teriam ocorrido em profundidades inferiores ao sistema radicular dos maiores indivíduos arbóreos que compõem a Floresta Ombrófila Densa, com mais de $3 \mathrm{~m}$ de profundidade. Dessa forma, o modelo TRIGRS indicou que a variação do FS para a sub-bacia em análise ultrapassa as superfícies de ruptura entre $1,5 \mathrm{~m}$ e $2 \mathrm{~m}$, considerada, em muitas pesquisas, comum para a Serra do Mar (Tabela 4), se aproximando mais da profundidade observada nos trabalhos de Cruz (1979) e Silveira et al. (2012).

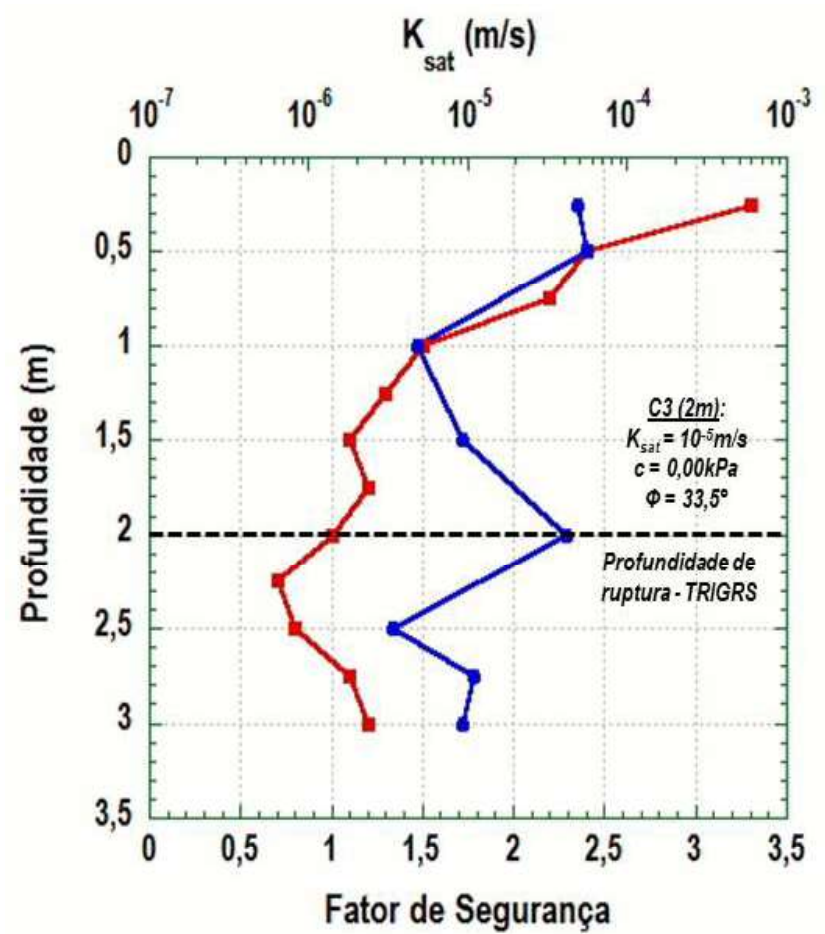

Figura 6 - Variação do Fator de Segurança (em vermelho) para a sub-bacia teste e da $K_{\text {sat }}$ (em azul) conforme a profundidade do solo. A cicatriz C3 encontra-se no perímetro da sub-bacia teste, onde a variação do FS indicou a superfície de ruptura aos $2 \mathrm{~m}$. Nesta profundidade, os valores obtidos para a C3 foram: $k_{\text {sat }}=10^{-5} \mathrm{~m} / \mathrm{s} ; \mathrm{c}=0,00 \mathrm{kPa}$ e $\phi=33,5^{\circ}$.

Tabela 3: Parâmetros de entrada do TRIGRS $\left(\boldsymbol{k}_{\text {sat }} ; \boldsymbol{c}\right.$ e $\left.\phi\right)$ em diferentes profundidades. Em vermelho, destacam-se as profundidades mais próximas da possível superfície de ruptura (entre $2 \mathrm{~m}$ e 2,5 m).

\begin{tabular}{c|c|c|c|c}
\hline Cicatriz & Profundidade (m) & $\boldsymbol{c}(\mathbf{k P a})^{*}$ & $\phi\left(^{\circ}\right)^{*}$ & $\boldsymbol{k}_{\text {sat }}(\mathbf{m} / \mathbf{s})^{*}$ \\
\hline \multirow{2}{*}{$\mathbf{C 1}$} & 0,50 & 0,00 & 29,1 & $10^{-4}$ \\
\cline { 2 - 5 } & $\mathbf{2 , 0 0}$ & $\mathbf{1 , 1 9}$ & $\mathbf{3 1 , 9}$ & $10^{-4}$ \\
\hline \multirow{2}{*}{ C2 } & 0,50 & 2,16 & 25,8 & $10^{-6}$ \\
\cline { 2 - 5 } & $\mathbf{2 , 0 0}$ & $\mathbf{1 1 , 0 5}$ & 36,8 & $10^{-5}$ \\
\hline \multirow{2}{*}{$\mathbf{C 3}$} & 0,50 & 0,00 & 36,7 & $10^{-5}$ \\
\cline { 2 - 5 } & $\mathbf{2 , 0 0}$ & $\mathbf{0 , 0 0}$ & 33,5 & $10^{-5}$ \\
\hline
\end{tabular}

*Fonte dos dados: Gomes (2012) e Ferreira (2013). 
Tabela 4: Valores de profundidade de instabilidade (superfícies de ruptura) obtidas em outras áreas experimentais da Serra do Mar.

\begin{tabular}{c|c|c|c|c}
\hline Local & $\begin{array}{c}\text { Superfície de } \\
\text { Ruptura }\end{array}$ & Litologia & $\begin{array}{c}\text { Forma de obtenção da } \\
\text { superfície de ruptura }\end{array}$ & Autor(es) \\
\hline $\begin{array}{c}\text { Serra do Mar, Cubatão } \\
\text { (SP) }\end{array}$ & $1,50 \mathrm{~m}$ e $2,0 \mathrm{~m}$ & Migmatitos & Em campo & $\begin{array}{c}\text { Wolle e Carvalho } \\
\text { (1989) }\end{array}$ \\
\hline $\begin{array}{c}\text { Serra do Mar, Rio de } \\
\text { Janeiro }\end{array}$ & $1,50 \mathrm{~m} \mathrm{e} 2,0 \mathrm{~m}$ & Gnaisses & Em campo & Campos et al. (1992) \\
\hline $\begin{array}{c}\text { Serra do Mar, Ubatuba } \\
\text { (SP) }\end{array}$ & $1,50 \mathrm{~m} \mathrm{e} 2,0 \mathrm{~m}$ & $\begin{array}{c}\text { Migmatitos e } \\
\text { granulitos }\end{array}$ & Em campo & Mendes (2008) \\
\hline $\begin{array}{c}\text { Bacia do Guaxinduba, } \\
\text { Serra do Mar (SP) }\end{array}$ & $\mathbf{2 , 0 0 ~} \mathbf{m ~ e ~} \mathbf{2 , 5 0 ~} \mathbf{~ m}$ & $\begin{array}{c}\text { Granitos e } \\
\text { gnaisses }\end{array}$ & Modelo TRIGRS & Este trabaho \\
\hline Serra do Mar, Paraná & $>3 \mathrm{~m}$ & $\begin{array}{c}\text { Granitos e } \\
\text { gnaisses }\end{array}$ & Em campo & Silveira et al. (2012) \\
\hline $\begin{array}{c}\text { Serra do Mar, } \\
\text { Caraguatatuba }\end{array}$ & $5 \mathrm{~m}$ & $\begin{array}{c}\text { Granitos, } \\
\text { gnaisses e } \\
\text { migmatitos }\end{array}$ & Em campo & Cruz (1979) \\
\hline
\end{tabular}

\section{Considerações Finais}

A maioria das pesquisas que utilizaram modelos matemáticos indicaram áreas de estabilidade e de instabilidade, muitas vezes com sucesso. Entretanto, esta pesquisa avançou as possibilidades de resultados apontados por modelos, ao apontar uma provável superfície de ruptura, exatamente na profundidade em que o FS atinge valores críticos ocasionando os escorregamentos na paisagem. Ademais, é importante destacar que este trabalho utilizou valores levantados in situ para a análise do Fator de Segurança com mais precisão na bacia. Dessa forma, os resultados indicaram uma superfície de ruptura hipotética mais profunda do que geralmente se considera para a Serra do Mar. Tal resultado pode ser importante para próximas pesquisas no que se refere ao planejamento de um futuro monitoramento hidrológico e geotécnico em tempo real, sobretudo para definir a localização mais precisa de instrumentos em campo, como sensores de umidade, por exemplo.

A relação específica da superfície de ruptura e de alguns parâmetros de entrada do TRIGRS pôde indicar a sensibilidade de algumas propriedades físicas dos solos na deflagração dos escorregamentos. Por exemplo, foi possível observar que os valores baixos de coesão foram comuns em duas das três cicatrizes avaliadas na potencial superfície de ruptura, conforme é consagrado pela literatura geomorfológica; assim como a presença de algumas descontinuidades hidráulicas. Em outras palavras, com a identificação de uma superfície de ruptura, avaliou-se a influência de determinado fator condicionante, sobretudo pedológico, na potencialização dos escorregamentos.

Por fim, os resultados obtidos pelo modelo podem ser utilizados pelo poder público, em suas diferentes esferas administrativas, para a definição e o monitoramento de áreas de risco e para o planejamento do uso da terra (ex. construção de moradias e de estradas, práticas florestais, entre outros).

\section{Agradecimentos}

Os autores agradecem ao $\mathrm{CNPq}$ (Edital Universal 480515/2011-5) e à FAPESP (Processo 2014/101092) pelo apoio financeiro de parte desta pesquisa. À EMPLASA (Contrato de Licença de uso CLU $\mathrm{N}^{\circ}$ 049/14) pela doação do MDT do município de Caraguatatuba; à Comissão Científica do XI Simpósio Nacional de Geomorfologia (Maringá, 2016) pela indicação de publicação na Revista Brasileira de Geomorfologia e aos pareceristas anônimos pelas alterações, sugestões e revisões.

\section{Referências Bibliográficas}

ALMEIDA, F. F. M.; CARNEIRO, C. D. R. Origem e evolução da Serra do Mar. Revista Brasileira de Geociências, v. 28, p. 135-150, 1998. 
AUGUSTO FILHO, O. Caracterização geológico-geotécnica voltada à estabilização de encostas: uma proposta metodológica. In: $1^{\text {a }}$. Conferência Brasileira de Estabilidade de Encostas (COBRAE). Rio de Janeiro. p. 721-733, 1992.

BAUM; R. L.; SAVAGE, W. Z.; GODT, J. W. TRIGRS: A Fortran Program for Transient Rainfall Infiltration and Grid-Based Regional Slope-Stability Analysis. U.S. Geological Survey Open-File Report, v. 2, 35 pp, 2002.

CAMPOS, J. L. E; VARGAS E. A. J; CAMPOS, T. M. P. Avaliação de campo da permeabilidade não-saturada de solos em encostas. In Conferência Brasileira sobre Estabilidade de Encostas - $1^{\circ}$ COBRAE, Rio de Janeiro, p. 371-382; 1992.

CHORLEY, R. J. Models in Geomorphology. In: CHORLEY, R. J.; HAGGETT, P. (Ed.). Models in Geography. Methuen \& Co., Londres, p. 43-96, 1967.

CROSTA, G. B.; FRATTINI, P. Distributed modelling of shallow landslides triggered by intense rainfall. Natural Hazards and Earth System Sciences, v. 3, p. 81-93, 2003.

CRUZ, O. A Serra do Mar e o litoral na área de Caraguatatuba: contribuição à geomorfologia litorânea. São Paulo, Série teses e monografias, n. 11. Tese (Doutorado em Geografia) - Instituto de Geografia - Universidade de São Paulo, 181pp., 1974.

DE PLOEY, Y.; CRUZ, O. Landslides in the Serra do Mar, Brazil. Catena, v. 6, p. 111-122, 1979.

EMPRESA PAULISTA DE PLANEJAMENTO METROPOLITANO - EMPLASA. Projeto de Atualização Cartográfica do Estado de São Paulo (Projeto Mapeia São Paulo). Empresa Paulista de Planejamento Metropolitano S/A - Emplasa; Diretoria de Planejamento -DPL e Unidade de Cartografia - UCA, 2011. (Contrato de Licença de uso CLU $\left.\mathrm{N}^{\circ} 049 / 14\right)$.

FERNANDES, N. F.; AMARAL, C. P. Movimentos de massa: uma abordagem Geológico-Geomorfológica. In: GUERRA, A.T. e CUNHA, S.B. Rio de Janeiro: Geomorfologia e Meio Ambiente. p. 123-194, 1996.

FERNANDES, N. F.; GUIMARÃES, R. F.; GOMES, R. A. T.; VIEIRA, B. C.; MONTGOMERY, D. R.; GREENBERG, H. Topographic controls of landslides in Rio de Janeiro: field evidence and modeling. CATENA, v.55, p. 163-181, 2004.

FERREIRA, S. F. Análise da influência das propriedades físicas do solo na deflagração dos escorregamentos translacionais rasos na Serra do Mar (SP). Dissertação (Mestrado em Geografia Física) - Faculdade de Filosofia, Letras e Ciências Humanas, Universidade de São Paulo. USP, São Paulo, 112 pp., 2013.

FÚLFARO, V. J.; PONÇANO, W. L.; BISTRICHI, C. A.; STEIN, D. P. Escorregamentos de Caraguatatuba: expressão atual e registro na coluna sedimentar da planície costeira adjacente. In: Congresso Brasileiro de Geologia de Engenharia 3, p.341350, 1976.

GAO, J. Identification of topographic settings conductive to landsliding from Nelson County, Virginia, U.S.A. Earth Surface Processes and Landforms, v. 18, p. 579-591, 1993.

GOMES, M. C. V. Análise da influência da condutividade hidráulica saturada dos solos nos escorregamentos rasos na bacia do rio Guaxinduba (SP). Dissertação (Mestrado em Geografia Física) - Faculdade de Filosofia, Letras e Ciências Humanas, Universidade de São Paulo. USP, São Paulo, 138 pp., 2012.

GOMES, M. C. V.; VIEIRA, B.C. Saturated hydraulic conductivity of soils in a shallow landslide area in the Serra do Mar, São Paulo, Brazil. Zeitschrift fur Geomorphologie, v. 60, p. 53-65, 2016.

GOMES, R.A.T. Modelagem de previsão de movimentos de massa a partir da combinação de modelos de escorregamentos e corridas de massa. Tese (Doutorado) - Universidade Federal do Rio de Janeiro, 102 pp., 206.

GUIMARÃES, R. F.; MONTGOMERY, D. R.; GREENBERG, H. M.; FERNANDES, N.F.; GOMES, R.A.T.; CARVALHO JÚNIOR, O. A. Parameterization of soil properties for a model of topographic controls on shallow landsliding: application to Rio de Janeiro. Engineering Geology, v. 69, p. 99-108, 2003.

GUZZETI, F., CARRARA, A; CARDINALI, M; REICHENBACH, P. Landslides hazard evaluation: a review of current techniques and their application in amulti-scale study, Central Italy. Geomorphology, v. 31, p. 181-216, 1999.

KIN, D.; IMA, S.; LEE, C.; WOOC, C. Modeling the contribution of trees to shallow landslide development in a steep, forested watershed. Ecological Engineering, v.61, p. 658-668, 2013.

LISTO, F. L. R.; VIEIRA, B. C. Influência de parâmetros geotécnicos e hidrológicos na previsão de áreas instáveis a escorregamentos translacionais rasos utilizando o modelo TRIGRS. Revista Brasileira de Geomorfologia, v. 16, p. 485$500,2015$.

LISTO, F. L. R.; VIEIRA, B. C. Mapping of risk and susceptibility of shallow-landslide in the city of São Paulo, 
Brazil. Geomorphology, v. 169-170, p. 30-44, 2012.

LOPES, E. S. S. Modelagem Espacial Dinâmica Aplicada ao Estudo de Movimentos de Massa em uma Região da Serra do Mar Paulista, na escala de 1:10.000. Tese (Doutorado) Universidade Estadual Paulista, Rio Claro, 302pp., 2006.

MENDES, R. M. Estudo das propriedades geotécnicas de solos residuais não saturados de Ubatuba (SP). Tese (Doutorado em Engenharia Civil) - Escola Politécnica da Universidade de São Paulo, 236pp., 2008.

MICHEL, G.P.; KOBIYAMA, M.; GOERL, R. F. Comparative analysis of SHALSTAB and SINMAP for landslide susceptibility mapping in the Cunha River basin, southern Brazil. Journal of Soils and Sediments, v.14, p. 1266-1277, 2014.

MONTGOMERY, D. R.; DIETRICH, W. E. A physically-based model for the topographic control on shallow landsliding. Water Resources Research, v. 30, p. 1153-1171, 1994.

MORGEnStern, N. R.; SANGREY, D. A. Methods of Stability Analysis. In: SCHURTER, R.L.; KRIZEK, R.J. (Eds.). Landslides: Analysis and Controls. TRB Special Reports, Washington, D.C. NAS-NRC, p. 155-171, 1978.

NERY, T. D.; VIEIRA, B. C. Susceptibility to shallow landslides in a drainage basin in the Serra do Mar, São Paulo, Brazil, predicted using the SINMAP mathematical model. Bulletin of Engineering Geology and the Environment (Print), v. 74, p. 369-378, 2014.

PACK, R. T.; TARBOTON, D. G.; GOODWIN, C. N. SINMAP - a stability index approach to terrain stability hazard mapping. User's manual. Terratech Consulting Ltd. Salmon Arm, B. C. Canada. 68 pp., 1998.

PERROTA, M. M.; SALVADOR, E. D.; LOPES, R. C.; D'AGOSTINO, L. Z.; PERUFFO, N.; GOMES, S. D.; SACHS, L. L.; MEIRA, V. T.; GARCIA, M. G. M.; LACERDA FILHO, J. V. Mapa Geológico do Estado de São Paulo. Escala 1:750.000. Programa Geologia do Brasil - PBG. CPRM, São Paulo, 2005.

RABACO, L. M. L. Avaliação de Modelos de Susceptibilidade a Movimentos Gravitacionais de Massa numa Faixa de Dutos. Dissertação de Mestrado. Universidade do Estado do Rio de Janeiro, Rio de Janeiro, 162pp., 2005.

SALCIARINI, D.; GODT, J. W.; SAVAGE, W.Z; CONVERSINI, P; BAUM, R.L; MICHAEL, J.L. Modeling regional initiation of rainfall-induced shallow landslides in the eastern Umbria Regional of Central Italy. Landslides, v. 3, p. 181-194, 2006.

SANTOS, D. D. As chuvas em Caraguatatuba (SP): Análise da variabilidade e eventos extremos em diferentes escalas temporais. Trabalho de Graduação Final (em Geografia), Departamento de Geografia, Faculdade de Filosofia Letras e Ciências Humanas, Universidade de São Paulo, 61pp., 2015.

SELBY, M. J. Hillslope: materials \& processes. Oxford University Press, New York, USA, edition 02, 480 pp., 1993.

SILVA, J. P.; SILVEIRA, C. T.; FIORI, A. P. Aplicação de dois métodos físico-matemáticos para avaliação do índice de estabilidade de vertentes da bacia do rio Ribeirão/ ParanaguáPR. Revista Geonorte, v. 2, p. 1291-1302, 2014.

SILVEIRA, C. T.; FIORI, A. P.; FERREIRA, A. M.; FELIPE, A. R.; KEPEL FILHO, J. L.; FOLADOR, R. M.; COSTA, L. C. Análise do fator de segurança da estabilidade das vertentes da bacia do rio Jacaré́, Serra do Mar Paranaense. Revista Brasileira de Geomorfologia, v. 13, p. 287-297, 2012.

SISTEMA INTEGRADO DE GERENCIAMENTO DE RECURSOS HÍDRICOS DO ESTADO DE SÃO PAULO - SIGRH; DEPARTAMENTO DE ÁGUAS E ENERGIA ELÉTRICA - DAEE (2015). Banco de Dados Hidrológicos. Disponível em: http://www.hidrologia.daee.sp.gov.br/. Último acesso em: julho/2015.

TERLIEN, M T. J.; ASCH, T. W. J.; VAN WESTEN, C. J. Deterministic modeling in GIS-Based landslide hazard assessment. In: CARRARA, A.; GUZZETTI, F. (Eds.): Geographical Information System in Assessing Hazards. Kluwer Academic Publishers, Dordrecht, p. 57-78, 1995.

van WESTEN, C. J. Geo-information tools for landslide risk assessment: an overview of recent development In Landslides: Evaluation and Stabilization. Taylor \& Francis Group, Rio de Janeiro, p. 39-53, 2004.

VARGAS J. E.; OLIVEIRA, A. R. B.; COSTA FILHO, L. M.; PRADO CAMPOS, L. E. A Study of the Relationship Between the Stability of Slopes in Residual Soils and Rain Intensity. In: International Symposium on Environmental Geotechnology. Envo Publishing, Leigh, U.S.A: p.491-500, 1986.

VIEIRA, B. C.; FERNANDES, N. F.; AUGUSTO FILHO, O. Shallow landslide prediction in the Serra do Mar, São Paulo, Brazil. Natural Hazards and Earth System Sciences (Print), v. 10 , p. 1829-2010, 2010.

VIEIRA, B. C.; FERREIRA, F. S.; GOMES, M. C. V. Propriedades físicas e hidrológicas dos solos e os escorregamentos rasos na Serra do Mar Paulista. RA'E GA: o Espaço Geográfico em Análise, v. 34, p. 269-287, 2015.

VIEIRA, B.C.; RAMOS, H. Aplicação do modelo SHALSTAB 
Listo F. L. R. et al.

para mapeamento da suscetibilidade a escorregamentos rasos em Caraguatatuba, Serra do Mar (SP). Revista do Departamento de Geografia da Universidade de São Paulo, v. 29, p. 172185, 2015.

WOLLE, C. M. Análise dos escorregamentos translacionais rasos numa região da Serra do Mar. Tese de Doutorado. Escola
Politécnica. Universidade de São Paulo, São Paulo, 406pp., 1988. WOLLE, C. M.; CARVALHO, C. S. Taludes Naturais. In: FALCONI, F.F.; JUNIOR, A. N. (Org). Solos do Litoral de São Paulo. São Paulo: ABMS, p. 180-203, 1994.

WOLLE, C. M.; CARVALHO, C.S. Deslizamentos em encostas na Serra do Mar - Brasil. Solos e Rochas, v. 12, p.27-36, 1989. 